\title{
Upregulation and Clinicopathological Significance of Long Non-coding NEAT1 RNA in NSCLC Tissues
}

\author{
Lin-Jiang Pan ${ }^{1 \&}$, Teng-Fei Zhong ${ }^{2 \&}$, Rui-Xue Tang ${ }^{2}$, Ping Li ${ }^{2}$, Yi-Wu Dang ${ }^{2}$, Su- \\ Ning Huang ${ }^{1}$, Gang Chen ${ }^{2 *}$
}

\begin{abstract}
Background: Recent reports have shown that nuclear enriched abundant transcript 1 (NEAT1), a long noncoding RNA (IncRNA), contributes to the precise control of gene expression and is related to several human malignancies. However, limited data are available on the expression and function of NEAT1 in lung cancer. The major objective of the current study was to profile the expression and clinicopathological significance of NEAT1 in non-small cell lung cancers (NSCLCs). Materials and Methods: NEAT1 expression in 125 NSCLC cases and paired adjacent non-cancer tissues was assessed by real-time quantitative reverse transcription-PCR (qRT-PCR). Relationships between NEAT1 and clinicopathological factors were also investigated. Results: The relative level of NEAT1 was $6.98 \pm 3.74$ in NSCLC tissues, significantly elevated as compared to that of the adjacent non-cancer lung tissues $(4.83 \pm 2.98, p<0.001)$. The area under curve (AUC) of high expression of NEAT1 to diagnose NSCLC was 0.684 (95\% CI: $0.619 \sim 0.750, p<0.001)$. NEAT1 expression was positively correlated with patient age $(\mathrm{r}=-\mathbf{2 . 0 0 7}, p=0.047)$, lymphatic metastasis $(\mathrm{r}=-2.731, p=0.007)$, vascular invasion $(\mathrm{r}=-3.617, p=0.001)$ and clinical TNM stage $(\mathrm{r}=-4.134, p<0.001)$. Conclusions: This study indicates that NEAT1 might be associated with oncogenesis and progression in NSCLC, and suggests application in molecular targeted therapy.
\end{abstract}

Keywords: NEAT1 - long non-coding RNA - NSCLC - RT-qPCR

Asian Pac J Cancer Prev, 16 (7), 2851-2855

\section{Introduction}

According to recent estimates, lung cancer remains as the leading cause of cancer-related deaths all over the world (Siegel et al., 2014), with an estimated 1.4 million deaths per year (Barrow and Michels, 2014). Non-small cell lung cancer (NSCLC) is the most frequent type of lung cancer, accounting for over $80 \%$ of all lung cancer cases (Chen et al., 2013d; Lee and Forey, 2013; Li et al., 2013). Despite recent diagnostic and therapeutic advancements, NSCLC is still deemed as the most aggressive malignant tumor with a frustrating 5-year overall survival rate as only 20-30\% (Ramshankar and Krishnamurthy, 2013; Rosell and Karachaliou, 2013). Thus, it is urgent to discover potential molecular targets of greater therapeutic values (Brothers et al., 2013).

Noncoding RNAs (ncRNAs), small ( $<200 \mathrm{~kb}$ ) and long (lncRNAs) (>200 kb), have attained extensive attention recently as the new crucial biological regulators from nuclear organization to epigenetic modification of posttranscriptional regulation and RNA splicing(Gupta et al., 2010; Tsai et al., 2010; Ma et al., 2012; Sen et al., 2014). Moreover, functional analyses have revealed that lncRNAs play essential roles in a range of developmental processes and diseases, including tumorigenesis and metastasis (Gupta et al., 2010; Cao, 2014; Liu et al., 2014). Aberrantly expressed lncRNAs are a hallmark of carcinomas, suggesting their potential application as predictive and prognostic biomarkers (Li et al., 2014; Liu et al., 2014). Nuclear enriched abundant transcript 1(NEAT1) is a nuclear-restricted lncRNA, which owns two isoforms: 3.7 kb NEAT1_1 and 23 kb NEAT1_2(Naganuma and Hirose, 2013). This lncRNA has been recently revealed to be an architectural component of a subnuclear structure called the paraspeckle, which is suggested to be involved in regulating gene expression by retaining mRNAs for editing in the nucleus (Chen and Carmichael, 2009). Anomalous NEAT1 expression has been reported in human malignancies, including of leukemia and ovarian carcinoma (Kim et al., 2010; Zeng et al., 2014). However, it is not yet fully understood how NEAT1 affects carcinogenesis and biological behavior of NSCLC. To date, only Zhou et al. (2014) found that in 5 metastatic lymph nodes from lung cancers, the NEAT1 was higher expressed than that in the original lung cancer tissues. No study has been carried out to elucidate the relationship between NEAT1 expression and the clinicopathological parameters in NSCLC. Further investigations are thus 
needed to establish the prognostic significance of NEAT1 in NSCLC.

In the current study, we evaluated the expression level of NEAT1 in 125 tumor tissues from patients with NSCLC as well as in their paired non-cancerous lung tissues. In addition, we analyzed the correlation of NEAT1 with a variety of clinicopathological parameters.

\section{Materials and Methods}

\section{Tissue samples}

The formalin-fixed, paraffin embedded (FFPE) tumor and paired adjacent non-cancerous lung tissues of 125 NSCLC patients (75 males and 50 females; mean age, 61.10 years; range, 23-90 years), who were admitted to the First Affiliated Hospital of the Guangxi Medical University (Nanning, Guangxi, China) between January, 2012 and February, 2014 were retrospectively evaluated in the current study.

The Ethical Committee of First Affiliated Hospital, Guangxi Medical University, China approved the current research, and informed consent was obtained from all participating patients. All samples were reviewed and diagnosed by two independent pathologists. The clinicopathological characteristics were summarized in Table 1. The EGFR status was detected as previously reported (Chen et al., 2010; Chen et al., 2011; Chen et al., 2012; Chen et al., 2013a; Chen et al., 2013b; Chen et al., 2013c).

Briefly, for EGFR protein expression detection, lung cancer tissue sections were de-paraffinized and antigen retrieval was performed with citrate buffer (pH.6.0) with $0.05 \%$ Tween-20. Slides were incubated with primary EGFR polyclonal antibody (sc-03, Santa Cruz Biotechnology, Inc., Santa Cruz, CA, USA) at $4^{\circ} \mathrm{C}$ overnight. The expression of EGFR protein was recorded with the quickscore ( $\mathrm{Q}$ score) based on the percentage $(\mathrm{P})$ of staining tumor cells $(0-100 \%)$ and the intensity (I) of staining ( 0 , complete absence of staining; 1 , faint cytoplasmic staining; 2 , moderate and incomplete membranous staining; 3 , strong membranous staining). The result of each case was scored by multiplying the percentage of positive tumor cells with the intensity $(\mathrm{Q}=\mathrm{P} \times \mathrm{I}$; maximum=3). A positive result was $\mathrm{Q}$ score $\geq 1$. Both the intensity and percentage of staining were assessed at low magnification (objective magnification $\times 10$ ), while the distribution of staining on membrane or cytoplasm, was evaluated at high magnification (objective magnification $\times 40$ ).

An overview of the IHC for all tissue sections was performed by two pathologists (Chen G and Dang Y). Two persons evaluated the staining results individualistically and inconsistencies in interpretation were resolved by consensus. Gene copy number per cell was investigated lung cancer tissue sections by FISH. The LSI EGFR Spectrum Orange/CEP7 Spectrum Green probe (Vysis, Abbott Laboratories, Illinois, USA) was used according to manufacturer instructions. FISH signals were evaluated under the fluorescence microscope Olympus BX41 (Olympus, Japan) equipped with single filters: DAPI, SpectrumOrange and FITC as well as a triple-filter DAPI/
FITC/SpectrumOrange. FISH analysis was independently performed by pathologists unaware of the clinical and molecular characteristics of patients. FISH negative NSCLCs were determined if with no or low genomic gain ( $\leq$ four copies of gene in $>40 \%$ of the cells) and FISH positive NSCLCs included gene amplification and high polysomy.

Gene amplification was defined by the presence of tight gene clusters, a gene/chromosome per cell ratio $\geq 2$, or $\geq 15$ copies of the genes per cell in $\geq 10 \%$ of the analyzed cells and high polysomy was identified as $\geq$ four copies of the gene in $\geq 40 \%$ of the cells. The QIAamp DNA FFPE Tissue Kit (Qiagen, Hilden, Germany) was used to extract DNA from paraffin-embedded tissues, and the operational tumor samples with histological control for the presence of tumor cells $(>75 \%)$ that was obtained by trimming the non-cancerous tissue and necrotic tissue. For mutational analysis of the kinase domain of EGFR coding sequence, exon 18, 19, 20, and 21 were amplified with specific pairs of primers, specific to the flanking sequences of individual exon with the EGFR reference sequence (NM_005228.3, NCBI). The assay was carried out according to the manufacturer's protocol with the ABI Step-one Plus real-time PCR system.

\section{$R T-q P C R$}

RNA isolation and RNA normalization were performed as described previously (Chen et al., 2013c; Rong et al., 2014). Reverse transcription (RT) and qPCR kits were applied to examine the expression of NEAT1 as reported before (Chen et al., 2013c; Rong et al., 2014). The primers for NEAT1 and reference gene GAPDH were as following: Neat1 Forward-5'-TGGCTAGCTCAGGGCTTCAG-3', Neat1 Reverse-5'-TCTCCTTGCCAAGCTTCCTTC-3'; GAPDH Forward-5'-TGAACGGGAAGCTCACTGG-3', GAPDH Reverse-5'-TCCACCACCCTGTTGCTGTA-3'. Real-time qPCR was performed with Applied Biosystems PCR7900. The NEAT1 expression was calculated with the formula $2^{-\Delta c q}$ (Chen et al., 2013c; Dang et al., 2014; Rong et al., 2014).

\section{Statistical analysis}

SPSS 20.0 (Munich, Germany) was used for statistical analysis. Student's t test was performed to analyze significance of difference between groups. Oneway analysis of variance (ANOVA) test was used to study the alteration of the expression levels of NEAT1 among different pathological grading and histological classification. Receiver operating characteristic (ROC) curves were generated to evaluate the power of NEAT1 to distinguish the NSCLC patients from non-cancerous lung tissue, as well as to predict some clinical features, including the status of lymph node metastasis, vascular invasion and clinical TNM stages. Survival analysis was assessed by the Kaplan-Meier method, and the log-rank test was applied to compare the survival time between groups. The risk factors for NSCLC were explored by using Cox proportional hazard regression model.

All $p$-values less than 0.05 , calculated by two-tailed test, were considered statistically significant, including with the aforementioned ANOVA test. 


\section{Results}

Upregulation and Clinicopathological Significance of Long Non-coding NEAT1 RNA in NSCLC

NEATlexpression in NSCLC tissues

The OD260/OD280 ratio of the total mRNA isolated from the FFPE tissues ranged from 1.85 to 2.05, and OD260/OD230 from 1.91 to 2.03. The PCR amplification efficiency of all the real time RT-qPCR reactions ranged from $91.3 \%$ to $95.5 \%$. The relative level of NEAT1 was $6.98 \pm 3.74$ in NSCLC tissues, significantly higher as compared to the adjacent non-cancerous lung tissues $(4.83 \pm 2.98, p<0.001$, Figure 1, Table 1). Furthermore,

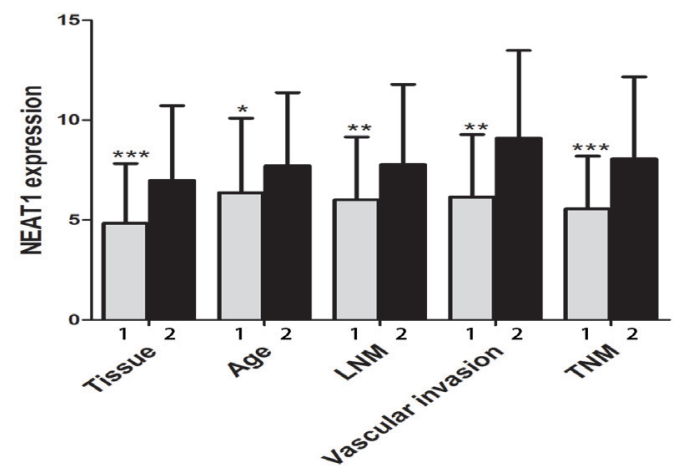

Figure 1. The Relationship between NEAT1 and Clinicopathological Parameters in NSCLC (Mean \pm SD). Tissue: 1 . adjacent non-cancerous lung; 2.NSCLC; patient age: 1 . $<=60$ years; 2 . $>60$ years; LNM (lymph node metastasis): 1 .No; 2.Yes; Vascular invasion: 1.No; 2.Yes; TNM: 1 . early stages (I and II); 2.advanced stages (III and IV). ${ }^{*} p<0.05 ; * * p<0.01$; $* * * p<0.001$
ROC curve was performed to assess the diagnostic contribution of NEAT1 in NSCLC. The area under curve (AUC) of NEAT1 was 0.684 (95\%CI: 0.619 0.750, $p<0.001$, Figure 2).

Relationship between the expression of NEAT1 and clinicopathological features in NSCLC

Concerning the correlation between NEAT1 expression and clinical features, NEAT1 was found to be related to several clinicopathological parameters. The relative expression of NEAT1 in patients whose age was older than

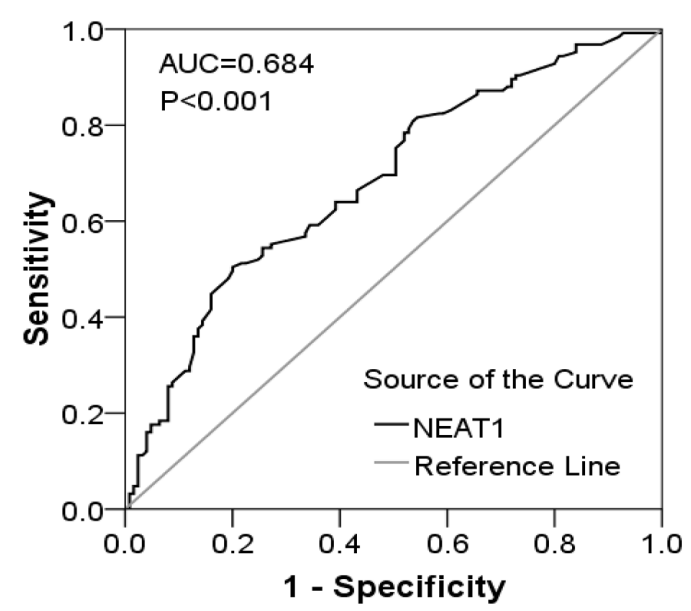

Figure 2. ROC Curve Analysis of NEAT1 for NSCLC. The area under curve (AUC) of NEAT1 was 0.684 (95\% CI: $0.619 \sim 0.750, p<0.001)$

Table 1. The Relationship between NEAT1 and Clinicopathological Parameters in NSCLC

\begin{tabular}{|c|c|c|c|c|c|}
\hline \multirow[t]{2}{*}{ Clinicopathological feature } & & \multirow[t]{2}{*}{$\mathrm{n}$} & \multicolumn{3}{|c|}{ NEAT1 relevant expression $\left(2^{-\Delta C q}\right)$} \\
\hline & & & $\overline{M e a n} \pm$ SD & $\mathrm{t}$ & P-value \\
\hline \multirow[t]{2}{*}{ Tissue } & Adjacent non-cancerous lung & 125 & $4.8342 \pm 2.9842$ & -7.966 & $<0.001$ \\
\hline & NSCLC & 125 & $6.9786 \pm 3.7447$ & & \\
\hline \multirow[t]{2}{*}{ Age (years) } & $<60$ & 57 & $7.7040 \pm 3.6739$ & 2.007 & 0.047 \\
\hline & $\geq 60$ & 68 & $6.3706 \pm 3.7214$ & & \\
\hline \multirow[t]{2}{*}{ Gender } & Female & 50 & $6.3532 \pm 3.5222$ & -1.533 & 0.128 \\
\hline & Male & 75 & $7.3956 \pm 3.8527$ & & \\
\hline \multirow[t]{2}{*}{ Smoke } & No & 38 & $6.3050 \pm 3.6297$ & -1.698 & 0.094 \\
\hline & Yes & 30 & $7.7637 \pm 3.3702$ & & \\
\hline \multirow[t]{2}{*}{ Tumor size $(\mathrm{cm})$} & $\leq 3$ & 60 & $7.0805 \pm 4.0064$ & 0.291 & 0.771 \\
\hline & $>3$ & 65 & $6.8846 \pm 3.5149$ & & \\
\hline \multirow[t]{2}{*}{ Lymph node metastasis } & No & 56 & $6.0125 \pm 3.1380$ & -2.731 & 0.007 \\
\hline & Yes & 69 & $7.7628 \pm 4.0268$ & & \\
\hline \multirow[t]{2}{*}{ Vascular invasion } & No & 90 & $6.1566 \pm 3.1195$ & -3.617 & 0.001 \\
\hline & Yes & 35 & $9.0926 \pm 4.3910$ & & \\
\hline \multirow[t]{2}{*}{ TNM } & I-II & 54 & $5.5583 \pm 2.6379$ & -4.134 & $<0.001$ \\
\hline & III-IV & 71 & $8.0589 \pm 4.1028$ & & \\
\hline \multirow[t]{3}{*}{ Pathological grading } & I & 17 & $6.4300 \pm 3.6075$ & $\mathrm{~F}=0.331^{\mathrm{a}}$ & 0.719 \\
\hline & II & 78 & $6.9538 \pm 3.6298$ & & \\
\hline & III & 30 & $7.3540 \pm 4.1802$ & & \\
\hline \multirow[t]{3}{*}{ Histological classification } & Adenocarcinoma & 101 & $6.7925 \pm 3.6424$ & $\mathrm{~F}=1.366^{\mathrm{a}}$ & 0.259 \\
\hline & Squamous carcinoma & 23 & $7.9517 \pm 4.1236$ & & \\
\hline & Large cell carcinoma & 1 & $3.4000 \pm 0$ & & \\
\hline \multirow[t]{2}{*}{ EGFR amplification } & No & 39 & $7.5272 \pm 3.3960$ & 1.317 & 0.193 \\
\hline & Yes & 18 & $6.2650 \pm 3.2869$ & & \\
\hline \multirow[t]{2}{*}{ EGFR protein expression } & Negative & 40 & $7.3480 \pm 3.5853$ & 0.748 & 0.458 \\
\hline & Positive & 17 & $6.6124 \pm 2.8909$ & & \\
\hline \multirow[t]{2}{*}{ EGFR mutation } & Wild type & 44 & $7.3275 \pm 3.5429$ & 0.814 & 0.419 \\
\hline & Mutation $^{\mathrm{b}}$ & 13 & $6.4554 \pm 2.8009$ & & \\
\hline
\end{tabular}

aANOVA; ${ }^{\mathrm{B} E G F R}$ mutation included 11 cases of short in-frame deletions in exon 19 and 2 cases of point mutations that result in a substitution of arginine for leucine at codon 858 (L858R) in exon 21 

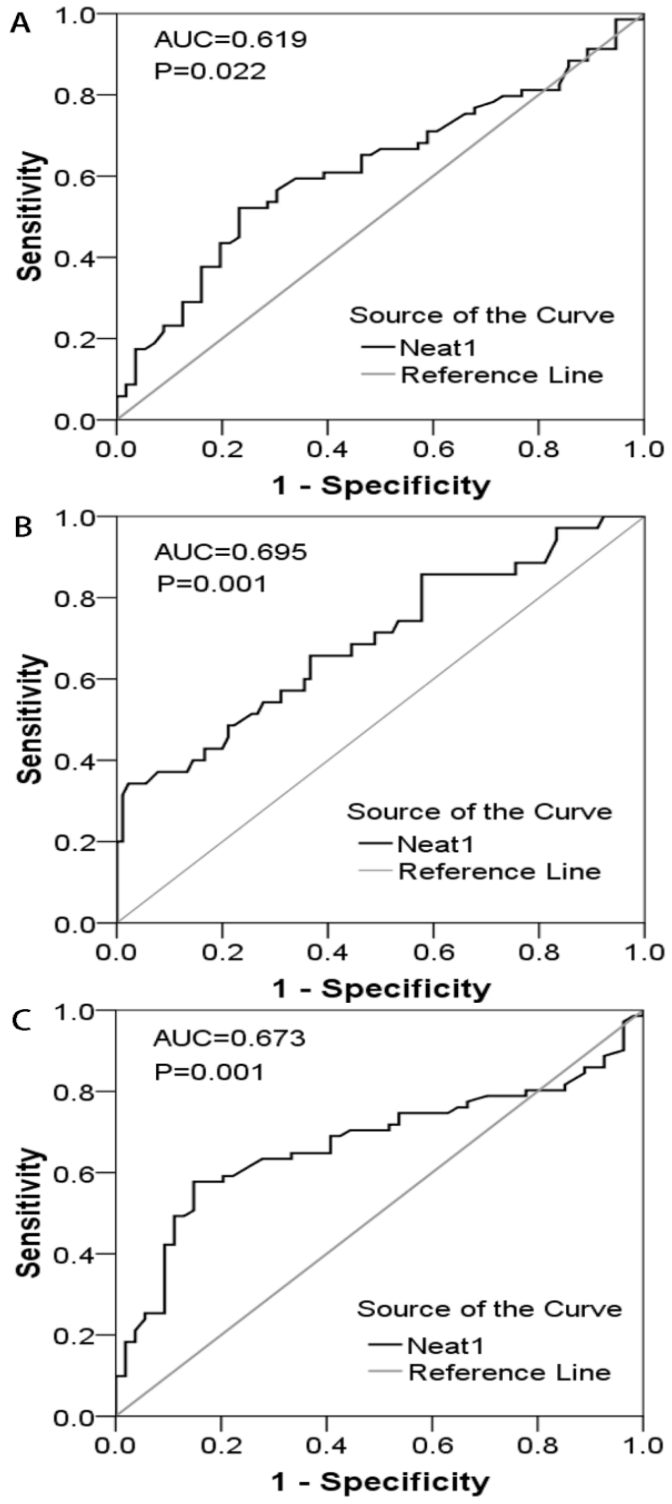

Figure 3. ROC Curve Analysis of NEAT1 for Clinicopathological Features. A: The area under curve (AUC) of NEAT1 was 0.619 for lymph node metastasis (95\% CI: 0.511 0.772, $p=0.046$ ); B: AUC was 0.695 for vascular invasion (95\% CI: 0.587 0.803, $p=0.001$ ); C: AUC was 0.673 for TNM (95\% CI: $0.577 \sim 0.768, p=0.001)$

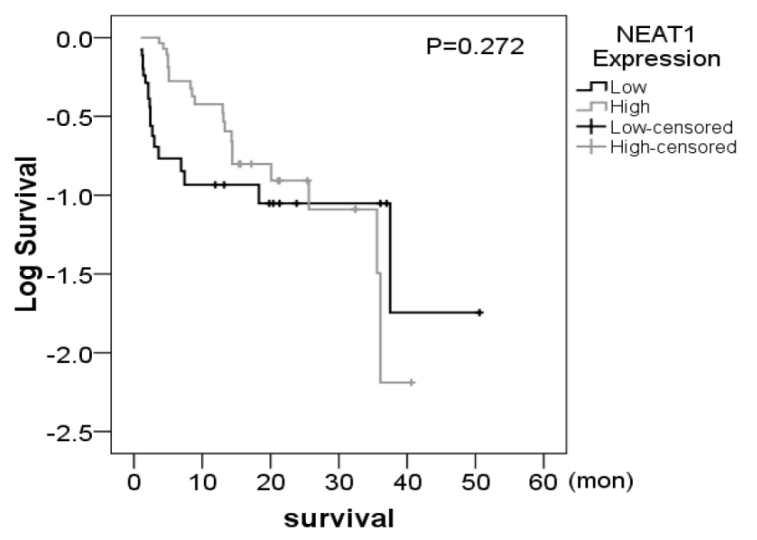

Figure 4. Correlation of NEAT1 Expression with Survival. There was no significant difference of survival between patients with low and high NEAT1 expression $(p=0.072)$
$60(6.3706 \pm 3.7214)$ was higher compared to that younger than $60(7.7040 \pm 3.6739, p=0.047)$. Higher level of NEAT1 was found in NSCLC patients with lymph node metastasis (7.7628 \pm 4.0268 ) compared with those without lymphatic metastasis $(6.0125 \pm 3.1380, p=0.007)$. Compared with those without vascular invasion $(6.1566 \pm 3.1195)$, the expression of NEAT1 was up-regulated in NSCLC patients with vascular invasion $(9.0926 \pm 4.3910$, $p=0.001$ ). Additionally, the relative expression of NEAT1 in advanced stages (III and IV, 8.0589 \pm 4.1028 ) was remarkably enhanced compared with that in early stages (I and II, 5.5583 $\pm 2.6379, p=0.004$, Table 1, Figure 1). Simultaneously, further analysis by Spearman correlation test showed the consistent relationship between NEAT1 expression and the following clinicopathological parameters: patient age $(\mathrm{r}=-0.197, p=0.027)$, lymph node metastasis $(\mathrm{r}=-0.205, p=0.022)$, vascular invasion $(\mathrm{r}=-0.303, p=0.001)$ and clinical TNM stages $(\mathrm{r}=-0.296$, $p=0.001)$. ROC curve was performed to identify the predictive value of NEAT1 level in NSCLC patients for clinicopathological features. ROC curve showed an AUC of 0.619 (95\% CI: $0.521 \sim 0.718, p=0.022$ ) to predict the status of lymphatic metastasis. As for the judgment of vascular invasion, the AUC in patients with vascular invasion was 0.695 (95\% CI: 0.587 0.803, $p=0.001$ ). Moreover, the AUC of ROC curve was 0.673 (95\% CI: $0.577-0.768, p=0.001)$ to evaluate the clinical TNM stage of NSCLC (Figure 3). However, no association was found between NEAT1 expression and other clinicopathological features, such as gender, smoke, tumor size, pathological grading, histological classification, EGFR amplification, EGFR protein expression, EGFR mutation status or survival. Among the 57 patients followed up, 29 had high NEAT1 expression (higher than the median level of NEAT1 6.4, while 28 had low expression. The survival time of high NEAT1 expression group was $14.400 \pm 10.803$ months, and it was $3.300 \pm 14.024$ months in low NEAT1 expression group. No significant difference was found between survival time and NEAT1 expression level $(p=0.272$; Figure 4).

\section{Discussion}

So far, there has been only one study which explored the role of NEAT1 in lung cancer by Zhao et al(Zhao et al., 2014). Five pairs of primary lung cancer and matched lymph node metastatic tissues were chosen to perform quantitative reverse transcriptase PCR analysis, in return they found out that HOTAIR and NEAT1 showed significant lower expression in primary lung cancer versus matched metastatic tissues $(p<0.01$ for HOTAIR and $p<0.05$ for NEAT 1 ). However, no study has been reported to investigate the distinction of NEAT1 expression between NSCLC and normal lung tissues. In the current study, we examined the NEAT1 expression in 125 cases of NSCLC samples and their paired non-cancerous lung tissues. We primarily found significantly higher expression of NEAT1 in NSCLC tissues. Additionally, ROC curve demonstrated that NEAT1 had a valid diagnostic value for NSCLC with the AUC of 0.684. NEAT1 could act as a tumor-promotional predictor in NSCLC. 
No study has been available regarding the relationship between expression of NEAT1 and clinicopathological parameters of NSCLC, except the report of Zhao et al with only 5 cases involved (Zhao et al., 2014). Zhao et al. (2014) found that NEAT1 expression was upregulated in the metastatic lymph node tissues compared to original lung cancer tissues. In the current study, a similar trend was observed that higher level of NEAT1 was detected in the NSCLC tissue with lymph node metastasis as compared with those without metastasis. Furthermore, NEAT1 expression was also related to patient age, status of vascular invasion and clinical TNM stage. The aforementioned results together with the literature indicated that NEAT1 may play a vital role in the progression and deterioration of NSCLC. However, no significant relationship was achieved between NEAT1 expression and patient survival. Further study with a larger patient sample size is required to clarify the prognostic value of NEAT1 in NSCLC patients. Zhao et al. (2014) also performed in vitro experiments to investigate the potential role and mechanism of NEAT1 in a metastatic lung cancer cell line NCI-H2009. By using migration assay, they failed to validate that knockdown of NEAT1 suppressed cell motility and invasion in cells, which was inconsistent with the findings from clinical tissues. The heterogeneity of cancer cells may partially explain the discordance. Different NSCLC cell lines need to be tested in vitro to explore the function and mechanism of NEAT1 on the phenotype of NSCLC cells.

In conclusion, IncRNANEAT1 may act as a oncogene, which plays an important role in the tumorigenesis and deterioration of human NSCLC. Further in vitro and in vivo studies are planned to explore the role and mechanism of NEAT 1 in the malignant phenotype of lung cancer cell lines.

\section{Acknowledgements}

The study was supported by the Fund of Guangxi Provincial Health Bureau Scientific Research Project (Z2013201,Z2014055) and the Fund of National Natural Science Foundation of China (NSFC 81360327). The funders had no role in study design, data collection and analysis, decision to publish, or preparation of the manuscript. Linjiang Pan and Tengfei Zhong contributed equally to this paper.

\section{References}

Barrow TM, Michels KB (2014). Epigenetic epidemiology of cancer. Biochem Biophys Res Commun. 455, 70-83.

Brothers JF, Hijazi K, Mascaux C, et al (2013). Bridging the clinical gaps: genetic, epigenetic and transcriptomic biomarkers for the early detection of lung cancer in the post-National Lung Screening Trial era. BMC Med, 11, 168.

Cao J (2014). The functional role of long non-coding RNAs and epigenetics. Biol Proced Online, 16, 11.

Chen G, Kronenberger P, Teugels E, et al (2011). Influence of RT-qPCR primer position on EGFR interference efficacy in lung cancer cells. Biol Proced Online, 13, 1 .

Chen G, Kronenberger P, Teugels E, et al (2012). Targeting the epidermal growth factor receptor in non-small cell lung cancer cells: the effect of combining RNA interference with tyrosine kinase inhibitors or cetuximab. BMC Med, 10, 28.

Chen G, Kronenberger P, Teugels E, et al (2013a). Effect of siRNAs targeting the EGFR T790M mutation in a non-small cell lung cancer cell line resistant to EGFR tyrosine kinase inhibitors and combination with various agents. Biochem Biophys Res Commun, 431, 623-9.

Chen G, Kronenberger P, Umelo IA, et al (2010). Quantification of epidermal growth factor receptor T790M mutant transcripts in lung cancer cells by real-time reverse transcriptase-quantitative polymerase chain reaction. Anal Biochem, 398, 266-8.

Chen G, Noor A, Kronenberger P, et al (2013b). Synergistic effect of afatinib with su11274 in non-small cell lung cancer cells resistant to gefitinib or erlotinib. PLoS One, 8, e59708.

Chen G, Umelo IA, Lv S, et al (2013c). miR-146a inhibits cell growth, cell migration and induces apoptosis in non-small cell lung cancer cells. PLoS One, 8, 60317.

Chen LL, Carmichael GG (2009). Altered nuclear retention of mRNAs containing inverted repeats in human embryonic stem cells: functional role of a nuclear noncoding RNA. Mol Cell, 35, 467-78.

Chen X, Liu Y, Roe OD, et al (2013d). Gefitinib or erlotinib as maintenance therapy in patients with advanced stage non-small cell lung cancer: a systematic review. PLoS One, 8, e59314.

Dang YW, Zeng J, He RQ, et al (2014). Effects of miR-152 on cell growth inhibition, motility suppression and apoptosis induction in hepatocellular carcinoma cells. Asian Pac J Cancer Prev, 15, 4969-76.

Gupta RA, Shah N, Wang KC, et al (2010). Long non-coding RNA HOTAIR reprograms chromatin state to promote cancer metastasis. Nature, 464, 1071-6.

Kim YS, Hwan JD, Bae S, et al (2010). Identification of differentially expressed genes using an annealing control primer system in stage III serous ovarian carcinoma. BMC Cancer, 10, 576.

Lee PN, Forey BA (2013). Indirectly estimated absolute lung cancer mortality rates by smoking status and histological type based on a systematic review. BMC Cancer, 13, 189.

Li G, Zhang H, Wan X, et al (2014). Long noncoding RNA plays a key role in metastasis and prognosis of hepatocellular carcinoma. Biomed Res Int, 2014, 780521.

Li SG, Chen HY, Ou-Yang CS, et al (2013). The efficacy of Chinese herbal medicine as an adjunctive therapy for advanced non-small cell lung cancer: a systematic review and meta-analysis. PLoS One, 8, 57604.

Liu JH, Chen G, Dang YW, et al (2014). Expression and prognostic significance of IncRNA MALAT1 in pancreatic cancer tissues. Asian Pac J Cancer Prev, 15, 2971-7.

Ma H, Hao Y, Dong X, et al (2012). Molecular mechanisms and function prediction of long noncoding RNA. ScientificWorld J, 2012, 541786

Naganuma T, Hirose T (2013). Paraspeckle formation during the biogenesis of long non-coding RNAs. RNA Biol, 10, 456-61.

Ramshankar V, Krishnamurthy A (2013). Lung cancer detection by screening - presenting circulating miRNAs as a promising next generation biomarker breakthrough. Asian Pac J Cancer Prev, 14, 2167-72.

Rong M, He R, Dang Y, et al (2014). Expression and clinicopathological significance of miR-146a in hepatocellular carcinoma tissues. Ups J Med Sci, 119, 19-24.

Rosell R, Karachaliou N (2013). Lung cancer: Maintenance therapy and precision medicine in NSCLC. Nat Rev Clin Oncol, 10, 549-50.

Sen R, Ghosal S, Das S, et al (2014). Competing endogenous RNA: the key to posttranscriptional regulation. Scientific World J, 2014, 896206.

Siegel R, Ma J, Zou Z, et al (2014). Cancer statistics. CA Cancer J Clin, 64, 9-29.

Tsai MC, Manor O, Wan Y, et al (2010). Long noncoding RNA as modular scaffold of histone modification complexes. Science, 329, 689-93.

Zeng C, Xu Y, Xu L, et al (2014). Inhibition of long non-coding RNA NEAT1 impairs myeloid differentiation in acute promyelocytic leukemia cells. BMC Cancer, 14, 693.

Zhao W, An Y, Liang Y, et al (2014). Role of HOTAIR long noncoding RNA in metastatic progression of lung cancer. Eur Rev Med Pharmacol Sci, 18, 1930-6. 